\title{
El precio de la vivienda en España tras el inicio de la crisis económica: un análisis empírico
}

\author{
TORRES-TELLEZ, JONATHAN \\ Universidad Isabel I (Burgos) \\ Correo electrónico: jonathantorrestellez@gmail.com \\ MONTERo SOler, AlBerto \\ Universidad de Málaga \\ Correo electrónico: alberto.montero@uma.es
}

\begin{abstract}
RESUMEN
Esta investigación tiene como objetivo analizar los factores que han influido en los niveles de precios de la vivienda en España tras el inicio de la crisis económica. Para ello se ha elaborado un panel de datos con efectos fijos que comprende 20082016 para 17 regiones españolas, que incluye variables del lado de la demanda y de la oferta. Los resultados indican que la demanda sigue siendo el principal impulsor de los precios para el caso español. Además, la oferta también está presionando al alza los precios, un síntoma que puede indicar la posible existencia de una burbuja inmobiliaria.
\end{abstract}

Palabras clave: mercado de vivienda, precio de la vivienda, panel de datos, España.

Clasificación JEL: R21; R31.

MSC2010: 62M10; 62P20; 91B99. 


\title{
Housing prices in Spain after the beginning of the financial crisis: An empirical analysis
}

\begin{abstract}
The objective of this research is to analyse the factors that have influenced on housing market in Spain after the beginning of the financial crisis. To this end, this investigation has used a fixed effect data panel covering from 2008 to 2016 for 17 Spanish regions, which has taken into consideration supply and demand variables. Our findings suggest that demand is still the main price driver for the Spanish case. In addition, supply keeps pushing prices up, a symptom that may show the possible existence of a housing bubble.
\end{abstract}

Keywords: housing market, housing prices, panel data, Spain.

JEL classification: R21; R31.

MSC2010: 62M10; 62P20; 91B99.

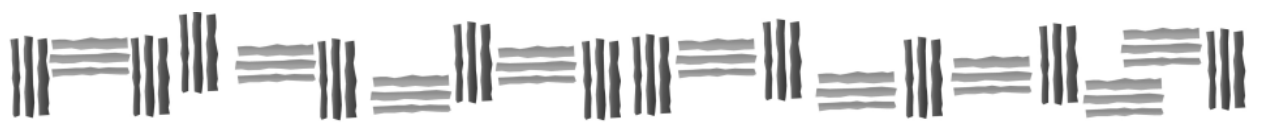




\section{Introducción.}

El sector inmobiliario ha sido uno de los principales pilares de la actividad económica en España durante las dos últimas décadas. Entre 1996 y 2007 creció a una tasa anual del 5\%, lo que ocasionó que el parque de vivienda aumentase en torno al 30\%, es decir, se edificaron 5,7 millones de viviendas convirtiendo a España en el país de la Unión Europea con más viviendas por cada mil habitantes (Arellano \& Bentolila, 2009).

Además, esta hipertrofia en la construcción de viviendas fue de la mano de una evolución desconocida en la historia reciente del precio de la vivienda en la mayor parte de economías occidentales. De este modo, los precios de la vivienda desde 1999 hasta 2008 tuvieron un importante crecimiento anual, lo que fue determinante para que en 20 años la vivienda se revalorizase en España un $232 \%$ (una vez descontada la inflación), e incluso hay fuentes que cifran este aumento del precio en más de un 300\% (Inurrieta, 2015).

Sin embargo, a finales de 2007 la situación tocó techo. La explosión de la crisis de las hipotecas subprimes en Estados Unidos produjo una sequía de los circuitos monetarios internacionales que golpeó directamente a la economía española. Todas las economías mundiales se vieron afectadas por este acontecimiento, pero en el caso de España la situación fue más grave debido a que para mantener esos ritmos de crecimiento había sido necesario un endeudamiento masivo. Es por ello que en el momento en el que el crédito exterior colapsó, se produjo un fallo en cadena dentro del sistema con el consiguiente derrumbamiento de la economía española (Naredo, 2010).

La crisis económica dio lugar a una fuerte corrección del sector, que no empezó a tener síntomas de recuperación hasta principios del 2014. Desde ese año la inversión real en vivienda ha tenido un crecimiento acumulado de un $45 \%$. No fue el único síntoma de la tendencia alcista que comenzaba a retomar el sector, ya que igualmente en estos últimos 6 años las transacciones de viviendas se han recuperado y han tenido un incremento de un $59 \%$ en valores acumulados, lo que las ha situado en torno a las 550.000 transacciones anuales (Alves \& Urtasun, 2019).

También los precios de la vivienda en España comenzaron una dinámica positiva y constante en el primer trimestre de 2014 tras una caída acumulada del 60\% en términos nominales que comenzó a finales de 2007 y que supuso alcanzar los precios más bajos de las dos últimas décadas. El crecimiento acumulado desde el momento del inicio de la recuperación de los precios hasta el 2018 alcanza el 27\% en términos nominales, aunque siguen siendo valores que están relativamente lejos de la sobrevaloración que alcanzó la vivienda en los años frenéticos de la burbuja inmobiliaria.

Gráfico 1. Índice de precio real de la vivienda (1T 2004=100).

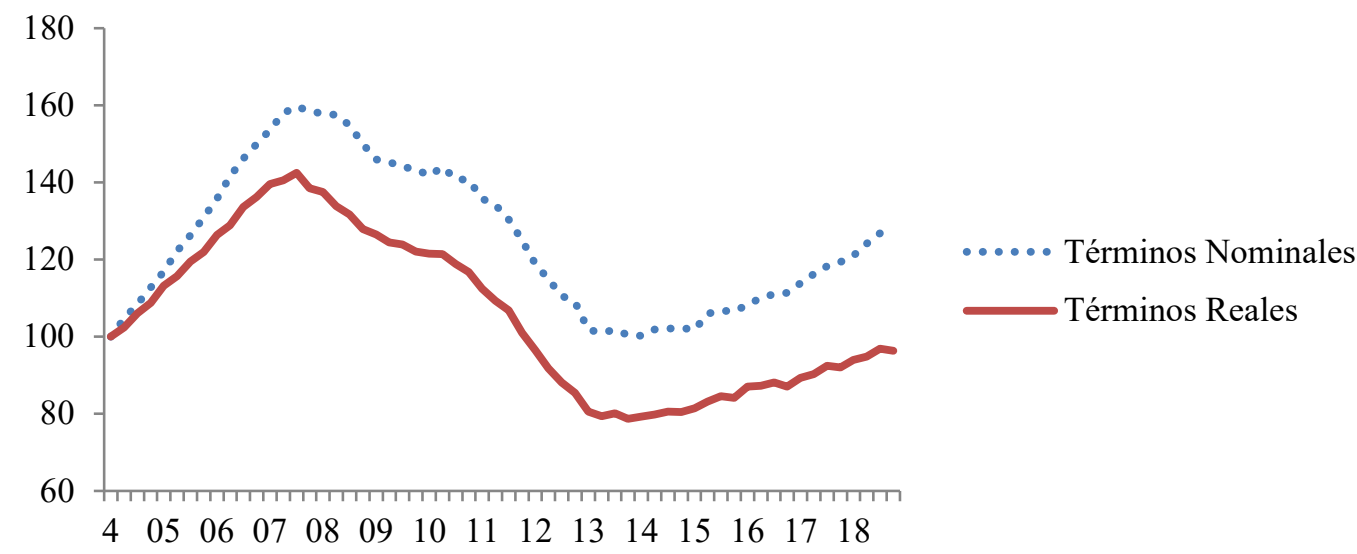

Fuente: Alves \& Urtasun (2019). 
Así, en un contexto de mejora del mercado de vivienda y un comportamiento alcista de los precios que se mantiene en los últimos años y con unos antecedentes tan alarmantes como los recientemente experimentados, esta investigación tiene como objetivo identificar determinados factores que pudieran haber influido en la evolución del precio de la vivienda en España después del inicio de la crisis económica. Para ello se ha analizado un panel de datos con efectos fijos para las regiones españolas que comprende el periodo 2008-2016 y que incluye tanto elementos desde el lado de la demanda como del lado de la oferta de vivienda.

La estructura de la investigación es la siguiente. A continuación, se hace un repaso de la literatura académica referida al tema. En la siguiente sección se describen las variables utilizadas y la estrategia empírica para analizar los datos. En el apartado 4 se examinan los principales resultados. Y, por último, se exponen las conclusiones.

\section{Estado de la cuestión.}

El precio de la vivienda ha sido un tema central de estudio en todos los países occidentales debido a los incrementos que ha experimentado en las últimas décadas y, sobre todo, por la crisis económica que tuvo lugar en 2007 tras la explosión de las hipotecas subprimes.

La mayor parte de los estudios han explicado el análisis de los precios de la vivienda a partir de la influencia que ejercen factores de demanda y de oferta en el mercado de la vivienda.

Entre los factores más determinantes por el lado de la demanda están los ingresos de la población y la evolución demográfica (Case \& Shiller, 2003). En la literatura existe consenso sobre que las fluctuaciones de los ingresos hacen variar en la misma dirección a la demanda y a los precios (Capozza et al., 2002; Gattini \& Hiebert, 2010; Maattanen \& Tervio, 2010).

Sin embargo, hay cierta controversia sobre la influencia de la población en los precios de la vivienda. Entre los estudios que defienden una correlación positiva destacan los de Jud y Winkler (2002), Catte et al. (2004) o Terrones y Otrok (2004). Mientras que hay otras investigaciones que afirman que el factor demográfico no es determinante, como Hilbers et al. (2008), Cvijanovic et al. (2010), o los trabajos de Glaeser et al. (2008) y Davidoff (2013) que señalan que para que esta variable sea decisiva es necesario que exista una oferta inelástica de vivienda.

Por el lado de la oferta también se ha elaborado una importante literatura. Distintos trabajos concluyen que el incremento de la oferta en dinámicas alcistas del mercado de vivienda puede conducir a la estabilización de sus precios (Glaeser \& Gyourko, 2006; Grimes \& Aitken, 2010; Caldera \& Johansson, 2013)

Otras investigaciones han analizados los efectos de diferentes componentes de la oferta sobre el precio de la vivienda, como la influencia del suelo urbanizable (Gyourko et al., 2008; Quigley et al., 2008), el impacto de las políticas de vivienda (Murray, 1999; Whitehead, 2003), o la sensibilidad de los precios a los tipos de interés (Iacoviello \& Minetti, 2003; Himmelberg et al., 2005; Adams \& Füss, 2010; Agnello \& Schuknecht, 2011).

Para el caso español también se han examinado los determinantes de los precios de la vivienda tanto por el lado de la oferta como por el lado de la demanda. Díaz-Fernández et al. (2018) analizan las relaciones entre el ciclo de la vivienda y la población y concluyen que existe un equilibro a largo plazo entre estas dos variables. También Taltavull (2003) y Taltavull y White (2012) determinan la existencia de una correlación positiva entre el precio de la vivienda y una serie de factores como la evolución demográfica o los ingresos para las principales ciudades españolas y para el caso comparativo entre España y Reino Unido, respectivamente. Además, el efecto positivo de los ingresos sobre el precio de la vivienda también ha sido afirmado por Fernández-Kranz y Hon (2006), Martínez y Maza (2003), 
Manrique y Ojah (2003) o Arestis y González-Martínez (2019) entre otros.

Desde el lado de la oferta Taltavull (2014) y Taltavull y Gabrielli (2015) analizan qué papel juega ésta en el precio en las regiones españolas y concluyen que existe una respuesta elástica de 2,5 entre estas dos variables. Las influencia de otros componentes de la oferta sobre el precio de la vivienda también han sido examinadas para este país: García-Montalvo (2010) investiga la incidencia del suelo en el precio de la vivienda y concluye que el aumento de los precios de los terrenos urbanizables es una consecuencia y no una causa del incremento del precio de la vivienda; Martínez y Maza (2003) parten de los elementos crediticios y afirman que tanto el tipo de interés nominal como el crecimiento del crédito a la vivienda explican una parte importante del aumento de los precios; o Cuestas (2017) que analiza los resultados de la entrada de capital extranjero y concluye que existe una retroalimentación entre éstos y los precios de la vivienda.

\section{Datos y modelo.}

Para analizar qué factores influyen en el precio de la vivienda en España, esta investigación ha empleado un panel de datos balanceado con observaciones anuales para 17 regiones que comprende el periodo 2008-2016 (se han excluido las ciudades autónomas de Melilla y Ceuta debido a la inexistencia de información sobre determinadas variables que emplea el estudio). Las series temporales no se han podido extender a los años 2017 y 2018 porque no se han publicado aún las cifras oficiales de algunas series para esas fechas.

Al utilizar datos a niveles regionales las unidades transversales que pueden ser objeto de estudio aumentan y, a su vez, los análisis pueden brindar relaciones más sólidas y estrechas entre las variables analizadas y el precio de la vivienda, como han evidenciado otros estudios (Quigley, 2007; Ball et al., 2010).

De esta forma, la variable dependiente empleada en esta investigación es el precio de la vivienda, que ha sido calculado como un índice de precios con base 2004 a partir del valor de las transacciones hechas cada año en las diferentes regiones de España.

En lo que respecta a las variables explicativas seleccionadas este estudio sigue la metodología de Wang y Kang (2014) y pone el foco en determinados factores del lado de la demanda y de la oferta. La Tabla 1 recoge todas las variables incorporadas al análisis de este trabajo.

Así, las variables que más inciden en las pautas del comportamiento de la demanda en el modelo neoclásico y que han sido seleccionadas son: los ingresos disponibles de las personas y la evolución demográfica (Case \& Shiller, 2003).

Por un lado, se asume que la población influye en los precios de la vivienda ya que un aumento de habitantes conllevará a la formación de un mayor número de hogares que presionará a la demanda de vivienda y, además, producirá una reducción de los recursos necesarios y disponibles que repercutirá en el costo de la misma (Jud \& Winkler, 2002).

Este factor fue muy importante por el lado de la demanda durante los años de la formación de la burbuja inmobiliaria debido al incremento de residentes que tuvo España y que supuso mayores requerimientos de vivienda (Taltavull, 2012). Es por ello que se considera necesario incluir este elemento en la investigación con el fin de analizar los efectos que tiene actualmente sobre el precio de la vivienda.

La capacidad adquisitiva de la sociedad española ha sido representada por el PIB per cápita. Esto es debido a que el precio de la vivienda en España ha estado relacionado con el ciclo económico en las últimas décadas, es decir, cuando el PIB per cápita ha tenido un comportamiento alcista el precio de la 
vivienda ha mantenido la misma dinámica, mientras que cuando se ha producido el empeoramiento de las condiciones económicas ha sido cuando los precios de la vivienda han caído (Fernández-Kranz y Hon, 2006). Además, la variación de los ingresos de la población puede suponer cambios en sus pautas de consumo y, por ende, afectar a la demanda, ya que muchos no propietarios de una vivienda, ante una mejora de sus rentas, pueden acudir al mercado con la intención de comprar una, a la vez que para las personas que ya son propietarias puede inducir el cambio a otra vivienda con mejores características, a la adquisición de una segunda residencia o a la inversión en bienes raíces (Wang \& Kang, 2014).

Tabla 1. Variables empleadas en la investigación.

\begin{tabular}{lccc}
\hline \multicolumn{1}{c}{ Nombre } & Definición & Unidad & Fuente \\
\hline Precio vivienda & Valor transacciones anuales & Índice Base=2004 & MIR \\
\hline Ingresos & PIB per cápita & Euros & INE \\
\hline Población & Población residente a 1 de enero & Personas & INE \\
\hline Inversión & FBCF en Construcción & Euros & Eurostat \\
\hline Precio $\mathrm{M}^{2}$ & Precio medio de $1 \mathrm{~m}^{2}$ & Euros & MIR \\
\hline Transacciones $\mathrm{M}^{2}$ & Área de venta anual & $\mathrm{M}^{2}$ & MIR
\end{tabular}

Fuente: Elaboración propia.

Mientras que por el lado de la oferta se han elegido las siguientes variables: la formación bruta de capital fijo (FBCF) en construcción, el precio del metro cuadrado y el total del área en las transacciones. La FBCF representa la inversión en construcción que se produce en cada región. Esta variable refleja, en gran parte, el estado del mercado de la vivienda a la vez que es la principal precursora de su desarrollo, ya que determina el ciclo de construcción, la velocidad del mismo y los costes necesarios para llevarlo a cabo (Wang \& Kang, 2014).

En este estudio se considera que este factor representa mejor el estado de la oferta del mercado de la vivienda español que el número de viviendas iniciadas, por ejemplo, ya que debido a la hipertrofia de la oferta en la época de la burbuja inmobiliaria los inicios de residencias personales han tenido un ligero retraso durante la recuperación del sector (Alves \& Urtasun, 2019). Además, hay que tener en cuenta que el proceso de construir una vivienda responde de una manera más lenta al estado del mercado debido a una serie de factores como el tiempo necesario entre el comienzo de una obra y acabarla, la información incompleta de la que se suele disponer sobre la situación del mercado y los requisitos financieros necesarios (Taltavull \& Gabrielli, 2015).

El precio del metro cuadrado hace referencia al suelo urbanizable. Este factor influye en los costos de la vivienda incrementando los costes de construcción y su escasez, derivado en parte de la dificultad para convertir terrenos no urbanizables en edificables, puede limitar la oferta de vivienda, lo que produce un desequilibrio entre la oferta y la demanda variando los precios de la vivienda (Gyourko et al., 2008). La relación esperada, por lo tanto, es que el precio del metro cuadrado de suelo urbanizable esté relacionado positivamente con los precios de la vivienda (Altuzarra \& Esteban, 2011).

Por último, los metros cuadrados totales de las transacciones realizadas cada año en las distintas regiones de España son utilizados como indicador de la circulación del mercado de vivienda puesto que refleja la demanda real del mismo. Esto se debe a que esta variable puede recoger en ocasiones mejor las fluctuaciones que experimenta el mercado de la vivienda que los precios, ya que al estar relacionado directamente con la oferta de vivienda un cambio en la demanda puede suponer una modificación en la provisión de viviendas (Wang \& Kang, 2014). 


\subsection{Especificación del modelo.}

Todas las variables descritas con anterioridad han sido analizadas usando un panel de datos con efectos fijos. La elección de este modelo se plantea de una manera más eficaz y razonable que el uso de otros métodos, como pueden ser los conjuntos de datos convencionales de secciones transversales o series de tiempo, debido a las características que presenta esta metodología (Hsiao \& Sun, 2000).

Entre las ventajas de seleccionar este modelo destaca la mayor capacidad informativa de los datos de panel, ya que permiten controlar la heterogeneidad individual de los datos, tener más grados de libertad y disminuir la colinealidad entre las variables explicativas, lo que proporciona estimaciones econométricas con mayor precisión (Hsiao, 2003). De esta manera, estas propiedades posibilitan supervisar en el modelo las diferencias existentes entre cada región de España como puede ser el tamaño poblacional, la inversión o el precio del suelo, lo que puede proporcionar relaciones estadísticas más sólidas y significativas.

Además, los paneles de datos tienen mejor disposición a la hora de identificar y medir efectos que no son detectables en secciones únicamente transversales o temporales (Baltagi, 2005). Gracias a esta singularidad al introducir estimaciones de efectos fijos en el panel de datos permite incluir las características diferentes y particulares inobservables de cada región que no varían en el tiempo y controlar los posibles cambios que son estables a lo largo de los años, como pueden ser las modificaciones en la legislación urbanística. De esta forma, la ecuación empleada es la siguiente:

$$
y_{i t}=\sum_{i=1}^{n} a_{t}+\sum_{i=1}^{n} \delta_{i}+\beta X_{i t}+\varepsilon_{i t}
$$

donde el índice $i$ y $t$ corresponden a la región y al año, $y_{i t}$ es el precio de la vivienda, $a_{t}$ es el efecto fijo del año, $\delta_{i}$ es el efecto fijo de la región, $X_{i t}$ son las variables explicativas descritas y $\varepsilon_{i t}$ es el término error.

De este modo, insertando las variables empleadas en el estudio en dicha ecuación se obtiene (2):

$$
\begin{gathered}
\text { Precio }_{i t}=\sum_{i=1}^{n} a_{t}+\sum_{i=1}^{n} \delta_{i}+\text { Población }_{i t}+\text { Ingresos }_{i t}+\text { Inversión }_{i t}+ \\
\text { Área de transacciones } \\
i t
\end{gathered}
$$

Antes de estimar la ecuación se ha comprobado que el modelo está correctamente calibrado y que es estable. Con este fin se han seguido las siguientes fases de la hoja de ruta que se recoge en la Figura 1.

Figura 1. Proceso de la derivación del modelo.

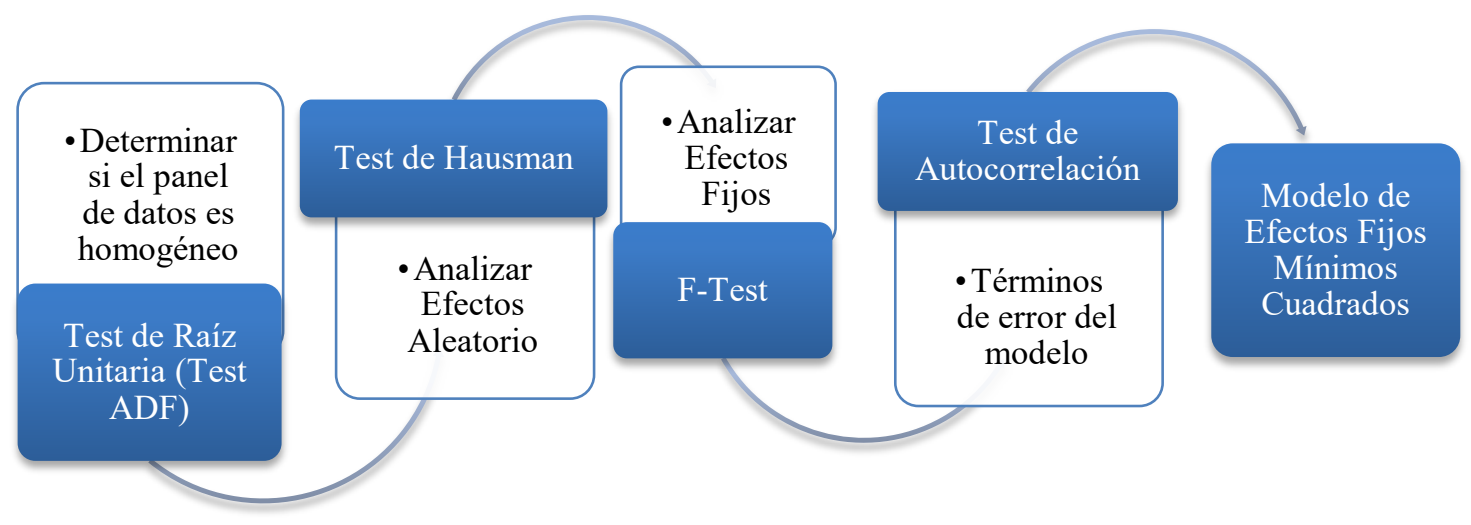

Fuente: Elaboración propia. 
El primer paso que se ha realizado ha sido aplicar el test de raíz unitaria ADF para confirmar que todas las variables empleadas en el estudio forman un panel de datos homogéneo.

Tabla 2. Resultados del Test ADF.

\begin{tabular}{llll}
\hline \multicolumn{1}{c}{ Variable } & \multicolumn{1}{c}{ Método } & Estadístico & Prob. \\
& & & \\
\hline Índice Precios & ADF - Fisher Chi-cuadrado & 103,896 & 0,000 \\
& ADF - Choi Z-estadística & $-6,71694$ & 0,000 \\
Transacciones m ${ }^{2}$ & ADF - Fisher Chi-cuadrado & 103,678 & 0,000 \\
& ADF - Choi Z-estadística & $-5,78261$ & 0,000 \\
Precio m ${ }^{2}$ & ADF - Fisher Chi-cuadrado & 66,1096 & 0,0008 \\
& ADF - Choi Z-estadística & $-3,82833$ & 0,0001 \\
PIB per Cápita & ADF - Fisher Chi-cuadrado & 89,3443 & 0,000 \\
& ADF - Choi Z-estadística & $-5,8477$ & 0,000 \\
Población & ADF - Fisher Chi-cuadrado & 59,3458 & 0,0046 \\
& ADF - Choi Z-estadística & $-3,54823$ & 0,0002 \\
Inversión & ADF - Fisher Chi-cuadrado & 117,914 & 0,000 \\
& ADF - Choi Z-estadística & $-7,47049$ & 0,000 \\
\hline
\end{tabular}

Nota: ADF, aumentado Dickey-Fuller.

Fuente: Elaboración propia.

En la segunda etapa se ha efectuado el test de Hausman para evaluar si en la regresión debían incluirse los efectos fijos o aleatorios del término error y de este modo comprobar si el modelo estadístico empleado se corresponde con los datos, ya que la inclusión de uno u otro componente puede dar resultados significativamente distintos (Wooldrige, 2002).

Tabla 3. Test de Hausman.

\begin{tabular}{lccc}
\hline \multicolumn{1}{c}{ Resumen del test } & $\begin{array}{c}\text { Chi-Cuadrado. } \\
\text { Estadístico }\end{array}$ & Chi-Cuadr. d.f. & Prob. \\
\hline Sección-transversal aleatoria & 0,00000 & 5 & 1,000 \\
Periodo aleatorio & 0,00000 & 5 & 1,000 \\
Sección-transversal y periodo aleatorio & 0,00000 & 5 & 1,000 \\
$*$ El test de varianza para la sección transversal no es válida. La estadística de Hausman es establecida en 0. & \\
$*$ El test de varianza para el periodo no es válida. La estadística de Hausman es establecida en 0. & \\
\hline
\end{tabular}

Fuente: Elaboración propia. 
La hipótesis nula de este test es que el uso de los efectos aleatorios es más correcto que el de los efectos fijos, por lo que un $\mathrm{p}$-valor $=1$ parece indicar que se debe emplear el modelo aleatorio ya que no se rechaza la hipótesis nula. Sin embargo, el test tanto para el periodo como para la sección transversal es 0 debido a que la prueba para la varianza es inválida. Estos resultados significan que no existen evidencias de efectos aleatorios individuales en los datos tanto en la sección transversal como en la temporal a pesar que los valores de p-valor puedan hacer pensar lo contrario.

Wooldrige (2002) señala que ante estos resultados muchos investigadores optan por el empleo de los efectos aleatorios, pero afirma que se puede estar cometiendo un error de Tipo II, es decir, no se puede rechazar el supuesto efectos aleatorios cuando éste es falso.

En otros trabajos ante esta situación, como Strat et al. (2016), rechazan la hipótesis nula del test de Hausman y emplean un modelo de efectos fijos que es más consistente tras realizar un F-test, ya que el test de Hausman no proporciona un resultado definitivo sobre qué modelo ha de emplearse.

De esta forma, se ha aplicado un F-test para probar la importancia de las estimaciones de los efectos fijos y comprobar si la inclusión de los mismos es correcta.

Tabla 4. F-Test.

\begin{tabular}{lccc}
\hline \multicolumn{1}{c}{ Test de efectos } & Estadístico & d.f. & Prob. \\
& & & \\
\hline Sección-transversal F & 17,488693 & $(16,123)$ & 0,0000 \\
Sección-transversal Chi-cuadrado & 181,504392 & 16 & 0,0000 \\
Periodo F & 16,923726 & $(8,123)$ & 0,0000 \\
Periodo Chi-cuadrado & 113,569601 & 8 & 0,0000 \\
Sección-transversal /Periodo F & 25,269332 & $(24,123)$ & 0,0000 \\
Sección-transversal /Periodo Chi-cuadrado & 272,359218 & 24 & 0,0000 \\
\hline
\end{tabular}

Fuente: Elaboración propia.

Como se puede comprobar en el Tabla 4 los valores obtenidos confirman que ni los efectos fijos individuales ni los temporales son redundantes, así como tampoco lo es la combinación de estos dos elementos. De esta forma, se ratifica que el empleo de los efectos fijos es más preciso que el uso de mínimos cuadrados ordinarios a la hora de considerar el término error en el modelo de regresión lineal de panel (Min \& Choi, 2009).

En la última fase se ha realizado el test de autocorrelación para el modelo de panel de datos de efectos fijos que se ha decidido emplear y se ha detectado un problema de autocorrelación de orden 1. Sin embargo, Min y Choi (2009) afirman que los modelos de efectos fijos no se ven afectados por la existencia de autocorrelación de orden 1 en el término de error, por lo que finalmente se confirma el uso de la ecuación (2) que se plasmó al inicio de este apartado.

\section{Resultados.}

Las estimaciones obtenidas tras aplicar el modelo de efectos fijos al panel de datos indican que cuatro de las cinco variables explicativas han tenido influencia en los precios tras el inicio de la crisis económica (Tabla 5). 
Tabla 5. Incidencia de los factores sobre la variable dependiente.

Variable Dependiente: Índice Precios

\begin{tabular}{lll}
\hline Inversión & 0,001133 & {$[0,000566]^{* *}$} \\
PIB per Cápita & 0,004252 & {$[0,001447]^{*}$} \\
Población & $4,03 \mathrm{E}-05$ & {$[1,71 \mathrm{E}-05]^{*}$} \\
Precio m ${ }^{2}$ & $-0,012873$ & {$[0,024209]$} \\
Transacciones $\mathrm{m}^{2}$ & 0,007694 & {$[0,002821]^{*}$} \\
$\mathrm{R}^{2}$ & 0,9 & \\
Número de obs. & \multicolumn{2}{c}{153} \\
\hline
\end{tabular}

Notas: SEs entre paréntesis. SEs robustos a heterocedasticidad y autocorrelación (Arellano, 1987).

$*, * *$ y *** indican significación al $1 \%, 5 \%$ y $10 \%$, respectivamente.

Fuente: Elaboración propia.

Los resultados por el lado de los factores de la demanda son claros y tanto la población como los ingresos tienen una relación positiva y significativa al $1 \%$ con los precios de la vivienda, lo que estaría en línea con los resultados de otras investigaciones (Case \& Shiller, 2003).

En el caso de la población el incremento de precios se produce debido a que al crecer el número de hogares el equilibro en el modelo de oferta y demanda se desplaza a un punto de la oferta agregada que se encuentra a niveles de precios superiores. De este modo, durante los primeros años de la crisis la población española se mantuvo constante e incluso llegó a descender, además los problemas económicos derivados de la crisis dificultaban la formación de nuevos hogares, una situación que coincidió con la caída de los precios (Gráfico 1), y es a partir del momento en que el número de habitantes ha comenzado a aumentar y se deja sentir la mejora económica cuando la demanda de vivienda se ha recuperado y ha incidido sobre los precios incrementándolos.

También se constata para el caso de España que los niveles de precios de la vivienda se han movido en la misma dirección que las modificaciones de los ingresos de la población tras la recesión económica ya que, según las estimaciones obtenidas, un aumento del PIB p.c. afecta positivamente a los precios de la vivienda. La explicación a esta correlación reside en que los precios de la vivienda no sólo reaccionan al número de personas que pueden acceder al mercado, sino que también es primordial que los niveles de ingresos de los hogares sean suficientes como para poder hacer frente a financiación para adquirir una vivienda (Taltavull, 2003). Además, cuando los niveles de renta son mayores las personas demandarán viviendas de mayor calidad y se facilita el acceso a la vivienda a un mayor número de personas, como pueden ser jóvenes o inmigrantes, que ante una mejor perspectiva laboral y/o de ingresos se plantean acudir a él, lo que repercutirá sobre su precio (Taltavull, 2003).

De esta forma, la situación económica de la población española empeoró durante los primeros años de la crisis lo que impidió a una gran parte de las familias acudir al mercado de vivienda, trasladándose esta disminución de la demanda a los precios. Sin embargo, en 2013 el PIB per cápita tuvo un crecimiento positivo tras media década de disminución y es justamente al año siguiente cuando el precio de la vivienda muestra síntomas de mejoría (Gráfico 1). En los años posteriores la renta de los hogares españoles ha seguido mejorando, al igual que el precio de la vivienda sigue con una dinámica alcista desde 2014, lo que confirmaría la estrecha relación entre ambos. 
En lo que respecta a las variables por el lado de la oferta también existe una relación positiva y significativa en el caso de la inversión y de las transacciones, mientras que el precio del suelo no tiene una relación estadísticamente significativa con el precio de la vivienda.

Los resultados muestran que el aumento de las transacciones en el mercado de vivienda español ha supuesto un incremento del precio de este bien raíz en los últimos años. Esto puede deberse a las expectativas de los participantes en el mercado que, ante la posibilidad de conseguir mayores beneficios económicos derivado de su transacción, tratan la compra de viviendas como una inversión esperando sacar rentabilidad a medio plazo (Capozza \& Seguin, 1996). De esta manera, la incorporación al mercado de las expectativas de mejores precios en el futuro supone un efecto potenciador de retroalimentación de los precios (Martínez \& Maza, 2003). Además, a esto se añadiría la aversión a las pérdidas de los propietarios que desean vender su vivienda para acceder a una mejor o nueva y que ante un mercado en auge, debido al incremento de transacciones, establecen precios por encima del mercado provocando un ajuste más lento de los mismos.

También puede influir que tras una mejora de los ingresos de la población después de un periodo de restricción de liquidez, como el ocurrido en España, aumente el volumen de las transacciones ante la posibilidad de cubrir la necesidad de nuevas viviendas de la población que no ha podido ser satisfecha en los últimos años debido a al estado de la económica. Esto hace que los precios crezcan hasta que se equilibre la situación como consecuencia de la inelasticidad de la oferta a corto plazo y de las expectativas positivas del mercado de vivienda que transmite confianza a los potenciales compradores a la hora de acudir a él (Arshanapalli \& Nelson, 2008).

La otra variable por el lado de la oferta que presenta una correlación positiva y significativa con los precios de la vivienda para el periodo analizado es la inversión. Este resultado es contrario a lo esperado atendiendo a la teoría económica, ya que en un contexto de precios al alza el aumento de la inversión debería producir un descenso de los precios debido a la mayor disponibilidad de oferta. Sin embargo, la relación que presentan estas dos variables podría apuntar a que se estén dando las condiciones para el inicio de una nueva burbuja inmobiliaria donde el incremento de la oferta no está repercutiendo sobre el precio. Esto podría ocurrir porque el rápido aumento de las ganancias derivadas de las operaciones de compra y venta en el mercado de vivienda en los últimos años hace más atractiva la inversión en este bien que en otros activos, por lo que el trasvase de un importante flujo de inversión hacia este mercado podría ayudar a que se formase una nueva burbuja de precios (Juárez, 2016).

Por último, los resultados para el precio del suelo muestran que esta variable no tiene incidencia sobre los niveles de precios para el caso español, lo que coincidiría con la fase de expansión anterior cuando los crecimientos de los precios se debieron a otros factores y no a la evolución del precio del suelo (García, 2010). La explicación sería que la demanda de suelo es una demanda derivada de la demanda de vivienda, por lo que el aumento de los precios de los terrenos urbanizables es una consecuencia y no una causa del incremento del precio de la vivienda (García, 2010).

Este resultado apoyaría las conclusiones de la teoría ricardiana, según la cual el valor del suelo es mayor en localizaciones más atractivas y donde los compradores estarían dispuestos a hacer mayores desembolsos, por lo que al aumentar la demanda de vivienda en esas zonas el precio del suelo se ve afectado en la misma dirección (Ooi \& Lee, 2006; Altuzarra \& Esteban, 2011).

Por último, se ha identificado en qué comunidades autónomas se prevé un índice de precios de la vivienda mayor a partir de las características propias inobservables de las mismas. Para ello se han estimado los efectos fijos individuales de cada región a partir del año 2014, debido a que es cuando en España el mercado inmobiliario mostró síntomas de mejoría tras la caída constante y continuada del precio desde 2009. 


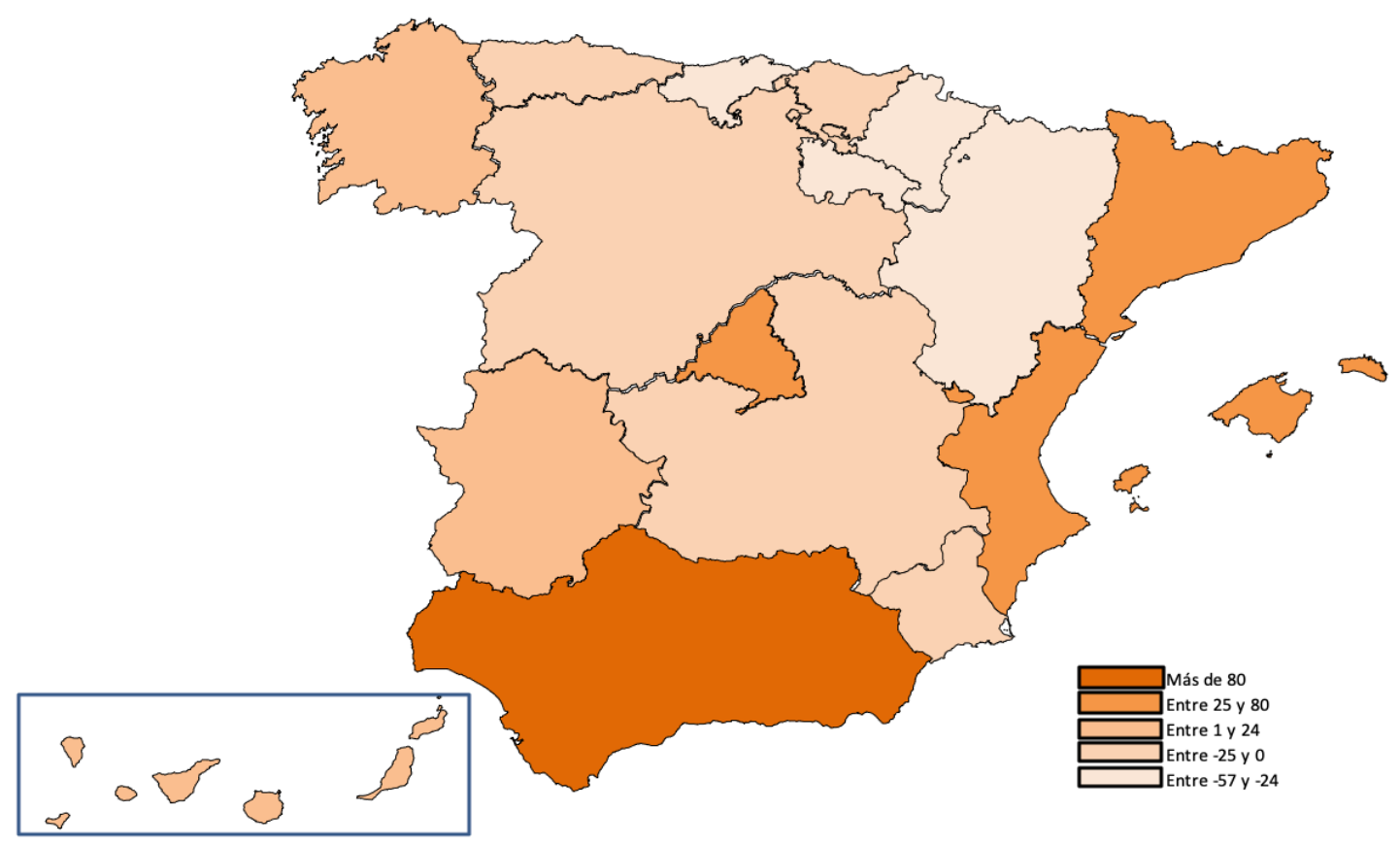

Fuente: Elaboración propia.

De este modo, se observa que Andalucía es la comunidad autónoma en la que, teniendo en cuenta sus particularidades propias, el precio de la vivienda puede crecer más ya que manteniendo constante el resto de las variables presenta un coeficiente de efectos fijos que supone un aumento por encima de los 80 puntos en el índice de precios.

Cataluña, la Comunidad de Madrid, la Comunidad Valenciana y las Islas Baleares son las otras regiones en las que, manteniéndose estables todas las variables del modelo, se esperarían unas subidas superiores del nivel de precios. De hecho, en esos territorios fue donde el valor promedio de las transacciones se recuperó más rápidamente tras la caída de 2008. En el caso andaluz, en el balear y en el valenciano fue impulsado por el importante peso que tiene el turismo en estas regiones, lo que ha favorecido la estimulación del mercado inmobiliario y el dinamismo de las transacciones. Y en lo que respecta a Cataluña y la Comunidad de Madrid se trata de las dos comunidades que tienen las ciudades más importantes de toda España y en las que el metro cuadrado construido alcanzó mayor valor antes de la crisis de 2008, con la excepción de Vizcaya en el País Vasco.

En el lado contrario se sitúan Navarra, Aragón, La Rioja y Cantabria, que son las comunidades en las que se prevé un menor índice de precios si se tienen en cuenta exclusivamente las características individuales de estas regiones. Esto coincide con el hecho de que se trata de los territorios en los que el mercado inmobiliario no terminó de experimentar una subida de precios en 2014, sino que ésta se retrasó durante un año, dando lugar a una recuperación no solo más tardía sino también más lenta.

Finalmente, es preciso destacar el caso del País Vasco, ya que a pesar de ser la Comunidad que registró un valor medio de las transacciones más elevado durante 2008-2016 y que, además, en 2014 también tuvo un crecimiento del precio de la vivienda; así, si se mantiene el resto de las variables constantes los efectos fijos individuales indican que el índice de precios descendería en 18 puntos. Este resultado es diferente al obtenido en el resto de comunidades que presentan circunstancias comunes, como la Comunidad de Madrid o Cataluña, por lo que parecería indicar que se han producido ciertas modificaciones en el mercado inmobiliario durante la recesión económica, un hecho que estaría amortiguando el incremento del precio. 


\section{Conclusiones.}

El mercado de vivienda en España está experimentando una notable mejoría en los últimos años, lo que ha supuesto la recuperación de los precios de la vivienda tras un lustro de caída continuada. Esta investigación intenta examinar los efectos de los determinantes teóricos en los niveles de precios después del inicio de la crisis económica. Para ello, se ha utilizado un panel de datos con efectos fijos para 17 regiones españolas que comprende el periodo 2008-2016 y en el que se han analizado la incidencia de factores tanto por el lado de la demanda como por el de la oferta sobre el precio de la vivienda

De esta manera, se confirma que la demanda sigue siendo un importante impulsor del precio de la vivienda en España, como ya ocurrió en la anterior fase de crecimiento de precios, ya que estos mantienen una relación positiva con las dos variables de la demanda examinadas, la evolución demográfica y los ingresos de la población.

Los factores de la oferta examinados también presionan los precios al alza, ya que tanto la inversión como las transacciones mantienen una correlación positiva y significativa, pero, sin embargo, al contrario de lo que muchas veces se piensa, no ocurre lo mismo con el precio del suelo. Esto último también coincidiría con los años expansionistas de la primera parte del siglo XXI cuando el aumento de los precios se debió a otros factores y no al suelo, y confirmaría que el incremento de los precios de los terrenos urbanizables es un resultado y no una causa del crecimiento del precio de la vivienda (García, 2010).

La incidencia positiva de las transacciones puede deberse a la incorporación de las expectativas de mejores precios en el futuro lo que supone un efecto potenciador de retroalimentación, ya que los participantes en el mercado ante la posibilidad de conseguir mayores beneficios económicos tratan la compra de la vivienda como una inversión esperando sacar rentabilidad a medio plazo.

Finalmente, la correlación obtenida para la inversión es preocupante, ya que siguiendo la teoría económica los resultados obtenidos deberían ser los contrarios, es decir, el incremento de la inversión en momentos en los que el precio está en una escalada positiva debería producir un descenso de los mismos debido a la existencia de una mayor disponibilidad de oferta. Sin embargo, esta relación no se cumple para el periodo analizado por lo que los valores obtenidos entre estas dos variables podrían señalar la existencia del inicio de una nueva burbuja de precios ya que el incremento de la oferta no está repercutiendo en el precio.

\section{Agradecimientos}

Agradecemos la sugerencia de uno de los revisores anónimos de hacer uso de los efectos fijos individuales.

\section{Referencias}

Adams, Z., \& Füss, R. (2010). Macroeconomic determinants of international housing markets. Journal of Housing Economics, 19, 38-50.

Agnello, L., \& Schuknecht, L. (2011). Booms and busts in housing markets: Determinants and implications. Journal of Housing Economics, 20(3), 171-190.

Altuzarra, A., \& Esteban, M. (2011). Land prices and housing prices: The case of Spain. Journal of Housing and the Built Environment, 26, 397-409. 
Alves, P., \& Urtasun, A. (2019). Recent housing market developments in Spain. Economic Bulletin, 2/2019, Banco de España.

Arellano, M. (1987). Computing robust standard errors for within-groups estimators. Oxford Bulletin of Economics and Statistics, 48, 431-434.

Arellano, M. \& Bentolila, S. (2009). La burbuja inmobiliaria: causas y responsables. En La crisis de la economía española: lecciones y propuestas, Libro electrónico Sociedad Abierta-Fedea.

Arestis, P., \& González-Martínez, A.R. (2019). Economic precariousness: A new channel in the housing market cycle. International Journal of Finances and Economics, 24(2), 1030-1043.

Arshanapalli, B., \& Nelson, W. (2008). A cointegration test to verify the housing bubble. The International Journal of Business and Finance Research, 2(2), 35-43.

Ball, M., Meen, G., \& Nygaard, C. (2010). Housing supply price elasticities revisited: evidence from international, national, local and company data. Journal of Housing Economics, 19(4), 255-268.

Baltagi, B.H. (2005). Econometric Analysis of Panel Data. Nueva York: John Wiley \& Sons Inc.

Caldera, A., \& Johansson, A. (2013). The price responsiveness of housing supply in OECD countries. Journal of Housing Economics, 22(3), 231-249.

Capozza, D., Hendershott, P., Mack, C., \& Mayer, C. (2002). Determinants of Real House Price Dynamics. NBER Working Paper 9262. Cambridge: National Bureau of Economic Research.

Capozza, D., \& Seguin, P. J. (1996). Expectations, efficiency, and euphoria in the housing martket. Regional Science and Urban Economics, 26(3-4), 369-386.

Case, K., \& Shiller, R. (2003). Is There a Bubble in the Housing Market? Brookings Papers on Economic Activity, 2, 299-362.

Catte, P., Girouard, N., Price, R. \& André, C. (2004). Housing markets, wealth and the business cycle. OECD Working Paper, N. 394.

Cuestas, J. (2017). House prices and capital inflows in Spain during the boom: Evidence from a cointegrated VAR and a structural Bayesian VAR. Journal of Housing Economics, 37, 22-28.

Cvijanovic, D., Favilukis, J., \& Polk, C. (2010). New in Town: Demographics, Immigration, and the Price of Real Estate. Londres: London School of Economics.

Davidoff, T. (2013). Supply Elasticity and the Housing Cycle of the 2000s. Real Estate Economics, 41(4), 793-813.

Díaz-Fernández, M., Llorente-Marrón, M., \& Méndez-Rodríguez, P. (2018). Interrelation between births and the housing market: A cointegration analysis for the Spanish case. Population, Space and Place, 25(3), e2172.

Fernandez-Kranz, D. \& Hon, M. (2006). A Cross-Section Analysis of the Income Elasticity of Housing Demand in Spain: Is There a Real Estate Bubble? Journal of Real Estate Finance and Economics, 32(4), 449-470.

García-Montalvo, J. (2010). Land use regulation and house prices in Spain. Moneda y Crédito, 230, 87-120. 
Gattini, L., \& Hiebert, P. (2010). Forecasting and Assessing Euro Area House Prices Through the Lens of Key Fundamentals. ECB Working Paper 1249. Frankfurt: European Central Bank.

Glaeser, E., \& Gyourko, J. (2006). Housing Dynamics. NBER Working Paper 12787. Cambridge, MA: National Bureau of Economic Research.

Glaeser, E., Gyourko, J. \& Saiz, A. (2008). Housing Supply and Housing Bubbles. Journal of Urban Economics, 64(2), 198-217.

Grimes, A., \& Aitken, A. (2010). Housing supply, land costs and price adjustment. Real Estate Economics, 38(2), 325-353.

Gyourko, J., Saiz, A., \& Summers, A. (2008). A new measure of the local regulatory environment for housing markets: the Wharton residential land use regulatory index. Urban Studies, 45(3), 693-729.

Hilbers, P., Hoffmaister, A.W., Banerji, A., \& Shi, H. (2008). House Price Developments in Europe: A Comparison. IMF Working Paper, 08/211. Washington: International Monetary Fund.

Himmelberg, C., Mayer, C., \& Sinai, T. (2005). Assessing high house prices: bubbles, fundamentals and misperception. Journal of Economic Perspectives, 19, 67-92.

Hsiao, C. (2003). Analysis of Panel Data. Cambridge. Cambridge: University Press.

Hsiao, C., \& Sun, B.H. (2000). To Pool or Not to Pool Panel Data, Panel Data Econometrics: Future Directions. En J, Krishnakumar and E. Ronchetti (eds.), Papers in Honor of Professor Pietro Balestra, Amsterdam: North Holland.

Iacoviello, M., \& Minetti, R. (2003). Financial liberalization and the sensitivity of house prices to monetary policy: theory and evidence. The Manchester School, 71, 20-34.

Inurrieta, A. (2015). Burbuja inmobiliaria y crisis en Andalucía: orígenes, dinámica y efectos. Universidad de Málaga.

Juárez, F. (2016). Vivienda y pobreza (Tesis doctoral). Universidad de Alicante, Departamento de Análisis Económico Aplicado.

Jud, D., \& Winkler, D. (2002). The dynamics of metropolitan housing prices. Journal of Real Estate Research, 23(1/2), 29-45.

Maattanen, N., \& Tervio, M. (2010). Income distribution and housing prices: an assignment model approach. CEPR Discussion Papers, 945. C.E.P.R.

Manrique, J., \& Ojah, K. (2003). The demand for housing in spain: an endogenous switching regression analysis. Applied Economics, 35(3), 323-336.

Martínez, J. \& Maza, L.A. (2003). Analysis of house prices in Spain. Working Paper Bank of Spain, $\# 0307$.

Min, I.S., \& Choi, P. S. (2009). Panel data analysis. Seoul, South Korea: The Korea Association of STATA.

Murray, M.P. (1999). Subsidized and unsubsidized housing stocks 1935-1987: crowding out and cointegration. The Journal of Real Estate Finance and Economics, 18(1), 107-124.

Naredo, J.M. (2010). El modelo inmobiliario y sus consecuencias. Boletín $C F+S, 44,13-27$. 
Ooi, J., \& Lee, S. (2006). Price discovery between residential land and housing markets. Journal of Housing Research, 15(2), 95-112.

Quigley, J.M. (2007). Regulations and property values in US: the high cost of monopoly. Working Paper W006-004, Berkeley Program on Housing and Urban Policy.

Quigley, J.M., Steven, R., \& Larry, R. (2008). Measuring land use regulations and their effects in the housing market. Working Paper W008-004, Berkeley Program on Housing and Urban Policy.

Strat, V.A., Davidescu, A.A., Grosu, R.M., \& Zgura, I.D. (2016). Regional Development Fueled by Entrepreneurial Ventures Providing KIBS - Case Study on Romania. Amfiteatru Economic Journal, 18(41), 55-72.

Taltavull, P. (2003). Determinants of housing prices in Spanish cities. Journal of Property Investment \& Finance, 21(2), 109-135.

Taltavull, P. (2012). The responsiveness of new supply to house prices: a perspective from the Spanish housing market. En C.M. Jones, M. White and N. Dunse (eds), Challenges of the Housing Economy: An International Perspective. London: Willey-Blackwell.

Taltavull, P. (2014). New housing supply and price reactions: Evidence from Spanish Markets. Journal of European Real Estate Research, 7 (1), 4-28.

Taltavull, P. \& Gabrielli, L. (2015). Housing Supply and Price Reactions: A Comparison Approach to Spanish and Italian Markets. Housing Studies, 30(7), 1036-1063.

Taltavull, P. \& White, M. (2012). Fundamental drivers of house price change: the role of money, mortgages, and migration in Spain and the United Kingdom. Journal of Property Research, 29(4), 341-367.

Terrones, M. \& Otrok, C. (2004). The global house price boom, IMF World Economic Outlook, September.

Wang, P. \& Kang, M. (2014). An empirical analysis on the housing prices in the Pearl River Delta Economic Region of China. International Journal of Urban Sciences, 18, 103-114.

Whitehead, C. (2003). The economics of social housing. En T. O'Sullivan, and K. Gibb, (eds), Housing Economics and Public Policy. London: Blackwell.

Wooldridge, J.M (2002). Econometric Analysis of Cross Section and Panel Data. Cambridge: MIT Press. 